\title{
Variable Properties in a Single Class of Excitatory Spinal Synapse
}

\author{
David Parker \\ Department of Zoology, University of Cambridge, Cambridge CB2 3EJ, United Kingdom
}

Although synaptic properties are specific to the type of synapse examined, there is evidence to suggest that properties can vary in individual synaptic populations. Here, a large sample of monosynaptic connections made by excitatory interneurons (EINs) onto motor neurons in the lamprey spinal cord locomotor network has been used to examine the properties of a single class of spinal synapse in detail.

The properties and activity-dependent plasticity of EIN-evoked EPSPs varied considerably. This variability occurred at convergent inputs made by several EINs onto single motor neurons. This suggests that it was an intrinsic network property and not simply related to differences between animals or experiments. The activity-dependent plasticity of EIN-evoked EPSPs could be negatively or positively related to the initial EPSP amplitude (P1 and P2 connections, respectively). This reflected the development of facilitation and depression from either small or large initial EPSPs.

To identify differences in presynaptic properties that could contribute to the synaptic variability, the quantal amplitude, release probability, number of release sites, and size of the available vesicle pool were examined. This analysis suggested that the variable amplitude and plasticity of EPSPs at P1 and P2 connections reflected an interaction between the release probability and the size of the available transmitter store.

There is thus significant functional variability in EIN synaptic properties. Synapses ranged from strong (evoked postsynaptic spikes) to weak (small depressing EPSPs). The selection of interneurons with different synaptic properties could provide an intrinsic mechanism for modifying excitatory network interactions and the locomotor network output.

Key words: interneuron; spinal cord; synaptic plasticity; neural network; motor neuron; lamprey

\section{Introduction}

The output of a neural network depends on the types of neurons it contains and the pattern of their synaptic connections ("network architecture"). To understand how a network output is generated, information is required on the cellular and synaptic properties of its component neurons (Selverston, 1980; Getting, 1989; Marder and Calabrese, 1996). Network cellular properties have been studied in some detail (Marder and Calabrese, 1996). Although the synapse is the site of information transfer, network synaptic properties have not been examined to the same extent. Potential synaptic influences on network activity include the sign, amplitude, and time course of PSPs, as well as activity-dependent changes in synaptic properties (Selverston, 1980; Getting, 1989).

Activity-dependent synaptic plasticity is a ubiquitous phenomenon. Its analysis has focused primarily on long-term effects associated with development and learning and memory (Byrne and Kandel, 1996; Feldman et al., 1999; Malenka and Nicoll, 1999). This plasticity can be considered as extrinsic, because it acts on the network to tonically alter its output. Plasticity could also develop during network activity to alter the strength of network synapses and thus act as an intrinsic mechanism for patterning rhythmic network activity (O'Donovan and Rinzel, 1997; Nadim and Manor, 2000; Fortune and Rose, 2001).

\footnotetext{
Received Dec. 2, 2002; revised Jan. 22, 2003; accepted Jan. 28, 2003

This work was supported by a Royal Society University Research Fellowship and grants from the Biotechnology and Biological Sciences Research Council and Wellcome Trust.

Correspondence should be addressed to David Parker, Department of Zoology, Cambridge University, Downing Street, Cambridge CB2 3EJ, UK. E-mail: djp27@cam.ac.uk.

Copyright $\odot 2003$ Society for Neuroscience $\quad 0270-6474 / 03 / 233154-10 \$ 15.00 / 0$
}

I have begun to examine intrinsic activity-dependent synaptic plasticity in the lamprey locomotor network (Parker, 2000a). This analysis requires information on the network architecture and the functional properties of network neurons. In particular, paired recordings must be made from identified presynaptic and postsynaptic neurons, and presynaptic cells must be stimulated at network-relevant spike frequencies. These requirements can be met to some extent in the lamprey locomotor network (Buchanan, 2001). As in other systems (Thomson, 2000), activity-dependent plasticity is specific to the type of synapse examined (Parker, 2000a). Connections made between specific classes of network interneurons will thus have characteristic activity-dependent effects when the network is active. The properties of individual connections can vary, however, which suggests that specific network synapses do not necessarily form homogenous functional units. Although this variability is an important component to our understanding of how synaptic properties contribute to the patterning of network activity, the small sample sizes obtained in previous analyses have prevented its relevance from being examined.

Here, a large sample of connections made by glutamatergic excitatory network interneurons (EINs) onto motor neurons has been used to examine the properties of this connection in detail. The results show considerable variability in the properties and plasticity of this single class of synapse. The variability was associated with differences in presynaptic release properties at different connections. The variability occurred at convergent inputs to single motor neurons. It thus cannot be accounted for by differences between animals or postsynaptic motor neurons, but in- 
stead represents an intrinsic property that could contribute to the patterning of the network output.

\section{Materials and Methods}

Adult male and female lampreys (Lamptera fluviatilis) were anesthetized with MS-222, and the spinal cord and notochord were removed. The spinal cord was isolated from the notochord and placed ventral side up in a Sylgard-lined chamber where it was superfused with Ringer's solution containing (in mM): $138 \mathrm{NaCl}, 2.1 \mathrm{KCl}, 1.8 \mathrm{CaCl}_{2}, 1.2 \mathrm{MgCl}_{2}, 4$ glucose, 2 HEPES, 0.5 L-glutamine. The Ringer's solution was bubbled with $\mathrm{O}_{2}$, and the $\mathrm{pH}$ was adjusted to 7.4 with $1 \mathrm{M} \mathrm{NaOH}$. The experimental chamber was kept at a temperature of $10-12^{\circ} \mathrm{C}$.

Paired recordings were made from EINs and motor neurons using thin-walled micropipettes filled with $3 \mathrm{M}$ potassium acetate and $0.1 \mathrm{M}$ potassium chloride. Motor neurons were identified by recording orthodromic extracellular spikes in the corresponding ventral root after current injection into their somata. EINs were identified by their ability to elicit monosynaptic EPSPs in motor neurons (Buchanan, 1993). These were identified by their reliability and constant latency after presynaptic stimulation at $20 \mathrm{~Hz}$ (Berry and Pentreath, 1976). To minimize potential differences attributable to the location of cells in different regions of the spinal cord, all experiments were performed in the rostral trunk region (i.e., the first 20 segments of the spinal cord immediately caudal to the last gill). To reduce the possibility of differences caused by the relative positions of cells, the EIN was either in the same segment or one segment rostral to the motor neuron. There were no consistent differences associated with EPSPs evoked by EINs in the same segment or one segment rostral to the motor neuron (my unpublished observations). Reticulospinal axons were recorded in the lateral region of the ventromedial column. Reticulospinal axons were identified by their conduction velocities of at least $2 \mathrm{~m} / \mathrm{sec}$ and by recording antidromic and orthodromic extracellular spikes on the caudal and rostral ends of the spinal cord. An Axoclamp 2A amplifier (Axon Instruments, Foster City, CA) was used for voltage recording and current injection. In all experiments, the membrane potential in control and in altered Ringer's solutions was kept constant by injecting depolarizing or hyperpolarizing current using single electrode discontinuous current clamp. Data were acquired, stored, and analyzed on computer using an analog-to-digital interface (Digidata 1200, Axon Instruments) and Axon Instruments software (pClamp 8).

Single EPSPs were evoked at $0.1 \mathrm{~Hz}$ to examine basic synaptic properties. No activity-dependent plasticity occurred at this frequency. EIN spikes were evoked either by injecting $1 \mathrm{msec}$ depolarizing current pulses of 10-60 nA or, where possible, on rebound from hyperpolarizing current pulses (2-5 msec, $1-5 \mathrm{nA})$ to avoid stimulation artifacts on the action potential. The plasticity of inputs during spike trains was examined by stimulating the presynaptic EIN at frequencies of 5, 10, and 20 $\mathrm{Hz}$. These frequencies are within the range reported for interneuron spiking during network activity (Buchanan and Cohen, 1982; Buchanan and Kasicki, 1995). Four to 20 spike trains were evoked at $30 \mathrm{sec}$ intervals at each frequency. These were averaged to determine the properties of the connection. In some cases the initial EPSPs in the trains were used as a measure of low-frequency-evoked inputs. Twenty spikes were evoked in each train. Network interneurons spike up to five times during network activity (Buchanan and Cohen, 1982; Buchanan and Kasicki, 1995). The initial part of the spike train allowed network-relevant plasticity to be examined, whereas the latter part of the train was used to examine the mechanisms underlying the plasticity. Calcium was reduced to 75 or $50 \%$ in low-calcium Ringer's solution and increased to 150 or $200 \%$ in highcalcium Ringer's solution.

EPSP amplitudes were measured as the peak amplitude above the baseline immediately preceding the spike. At the frequencies used there was little summation of EPSPs during spike trains. The initial EPSP, the paired-pulse (PP) plasticity, and the plasticity over the 2nd-5th spikes $\left(\operatorname{Train}_{2-5}\right)$, the 6th-10th spikes $\left(\operatorname{Train}_{6-10}\right)$, and the 11th-20th spikes in the train $\left(\operatorname{Train}_{11-20}\right)$ were measured. Paired-pulse plasticity was expressed as $\mathrm{EPSP}_{2} / \mathrm{EPSP}_{1}$, and plasticity over different regions of the spike train was expressed as $\mathrm{EPSP}_{\text {Train }} / \mathrm{EPSP}_{1}$. Low-frequency-evoked EPSPs or the initial EPSPs in the trains were used to measure EPSP rise times, amplitudes, and half-widths. Where stimulation artifacts did not obscure
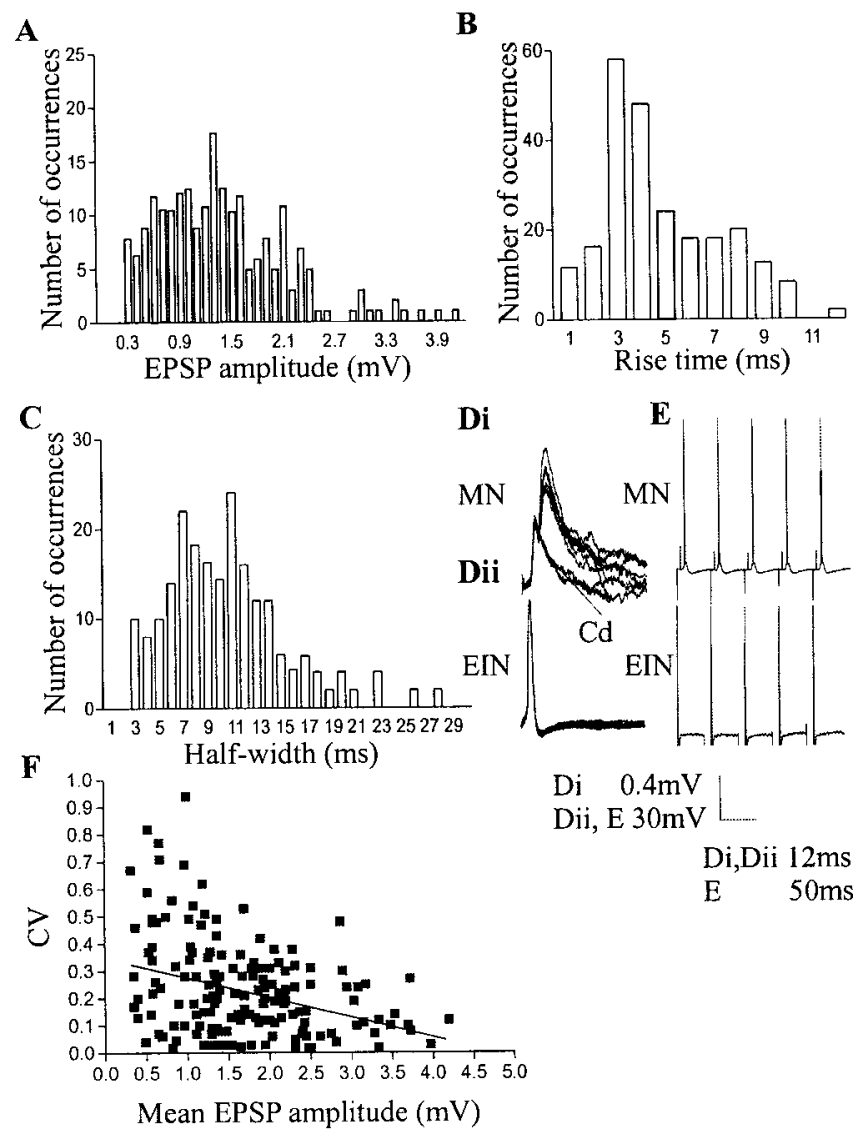

Di $0.4 \mathrm{mV}$

Dii, E $30 \mathrm{mV}$

Di,Dii $12 \mathrm{~ms}$

E $50 \mathrm{~ms}$

Figure 1. The properties of EIN-evoked EPSPs. Histograms of EPSP amplitudes $(A)$, rise times $(B)$, and half-widths $(C)$ are shown. Di, Dii, An example of an electrical connection between an EIN and a motor neuron. Notice that the chemical component varies, but the electrical component is constant. The chemical but not electrical component was blocked by cadmium (200 $\mu \mathrm{M})$. $E$, An example of a connection in which EIN stimulation at $20 \mathrm{~Hz}$ consistently evoked spikes in the postsynaptic motor neuron. $F$, The relationship of the coefficient of variation to the initial EPSP amplitude. Each symbol represents a single connection.

action potentials, their rise times, peak amplitudes, and half-widths were also measured.

Statistical significance was examined using two-tailed paired or independent $t$ tests or one-way ANOVA. When an ANOVA was used, a Tukey test was used for post hoc analysis of differences between groups. $n$ in the text refers to the number of connections examined. Up to seven connections were examined in a single animal ( $n=163$ animals). All values given refer to mean \pm SEM.

\section{Results}

\section{Properties of low-frequency-evoked EPSPs}

This analysis is based on a sample size of 278 monosynaptic EIN connections to motor neurons. In some connections $(n=26)$, polysynaptic excitatory and inhibitory inputs reliably followed the evoked EPSP. These connections have been examined separately and are not included in this analysis (my unpublished observations).

The mean amplitude of low-frequency-evoked EPSPs was $1.5 \pm 0.05 \mathrm{mV}$ (range, 0.32 to $4.2 \mathrm{mV}$ ) (Fig. $1 A$ ). The mean rise time was $3.7 \pm 0.18 \mathrm{msec}$ (range, $0.6-11.9 \mathrm{msec} ; n=100$ ) (Fig. $1 B$ ), and the mean half-width was $10.2 \pm 0.4 \mathrm{msec}$ (range, $3.4-$ $27.5 \mathrm{msec} ; n=100$ ) (Fig. $1 C$ ). These rise times and half-widths resemble those of non-NMDA-mediated and mixed NMDA and non-NMDA-mediated EPSPs in the lamprey (Dale and Grillner, 1986). There was no correlation between the EPSP amplitude and rise time $\left(r^{2}=0.01\right)$. Slower rise times were associated with 
longer half-widths in some cases $\left(r^{2}=\right.$ 0.28 ), possibly reflecting asynchronous transmitter release from several sites (Juttner et al., 2001). A moderate correlation between the EPSP amplitude and halfwidth $\left(r^{2}=0.35\right)$ may reflect the influence of the NMDA component on the EPSP. An inflection on the rising phase of the EPSP suggestive of an electrical component was present in 38 of 252 connections (15\%) (Fig. 1 Di,Dii). The presence of an electrical connection was verified in five of these connections by the depolarization that persisted after blocking chemical transmission with cadmium $(200 \mu \mathrm{M})$ (Fig. $1 D)$.

In a small proportion of connections $(n=8)$, EIN-evoked EPSPs reliably evoked spikes in the postsynaptic motor neuron (Fig. 1E). All cells were held at resting potentials of between -65 and $-70 \mathrm{mV}$, and thus spiking was not caused by a relatively depolarized membrane potential in some motor neurons. The EPSP amplitude, measured by hyperpolarizing the motor neuron by $10-20 \mathrm{mV}$ to prevent spiking, was significantly larger at connections in which spikes were evoked $(2.1 \pm$ $0.20 \mathrm{mV} ; p<0.05 ; n=6)$ than the overall mean EPSP amplitude $(1.5 \pm 0.05 \mathrm{mV})$. However, this could reflect the influence of the relatively hyperpolarized membrane potential on the EPSP. Also, because EPSPs larger than $2.1 \mathrm{mV}$ did not necessarily evoke spikes, the EPSP amplitude alone cannot account for the spiking. There was no significant difference in the rise times of EPSPs that did or did not evoke spikes $(3.2 \pm 0.7 \mathrm{msec}, n=6$, and $3.7 \pm 0.18$ msec, $n=100$, respectively), and thus the inputs presumably did not differ in their location relative to the postsynaptic spikeinitiating zone. In one experiment, spikes were evoked by two of three EINs that converged onto a single motor neuron. Because the probability of finding EINs that evoked spikes was low $(\sim 3 \%)$, this suggests that spiking reflected a property of the postsynaptic motor neuron.

Although their basic properties varied, a constant property of EIN inputs to motor neurons was that they were very reliable; EPSP failures were absent in normal Ringer's solution (my unpublished observations). Although EPSPs did not fail, the amplitude of successive low-frequency-evoked EPSPs could fluctuate. The coefficient of variation (CV) (SD/mean) of low-frequencyevoked EPSPs ranged from 0.06 to $0.94(n=169)$. EPSPs of $>1.5 \mathrm{mV}$ had lower CVs $(0.14 \pm 0.05)$ than EPSPs of $<1.5 \mathrm{mV}$ $(0.27 \pm 0.07)$, although the variability, particularly of small EPSPs, weakened the relationship between the EPSP amplitude and $\mathrm{CV}\left(r^{2}=0.12\right)$.

\section{Plasticity over spike trains}

In a previous analysis of the activity-dependent plasticity of EIN inputs to motor neurons, EPSPs usually depressed over spike trains (Parker and Grillner, 2000). However, the onset of depression over the train could vary, and facilitation occurred in a proportion of connections. The small sample size $(n=27)$ (Parker and Grillner, 2000) prevented the significance of this variability from being examined. This has now been possible with the large number of connections obtained here.
B

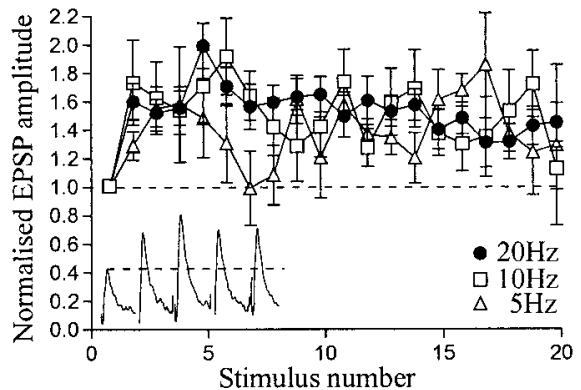

D

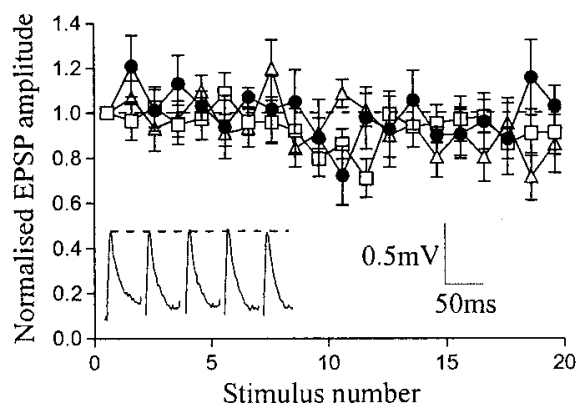

Figure 2. The plasticity of EIN inputs over trains of 20 spikes at $5-20 \mathrm{~Hz}$. Graphs show summed data at depressing $(A)$, facilitating $(B)$, biphasic $(C)$, and unchanged connections $(D)$. Insets on the graphs show examples of each type of plasticity over the first five spikes during a $20 \mathrm{~Hz}$ spike train.

There were four responses over spike trains: depression, facilitation, biphasic, and unchanged (no significant plasticity over the train) (Fig. 2). As in the previous analysis, depression was the usual effect at $20 \mathrm{~Hz}$, where it was seen in 103 of 225 connections (46\%). The depression reached a plateau at $\sim 60 \%$ of control between the 10th and 20th spikes in the train. Facilitation occurred in 61 connections (27\%). It developed to its maximum level of $\sim 160 \%$ of control by the second spike in the train. It was maintained at this level over $\operatorname{Train}_{6-10}$, but some recovery (between 10-20\%) occurred later in the train. Biphasic responses ( $n=33$ of 225 ) consisted of an initial facilitation to $\sim 120 \%$ of control over Train $2-5$ and depression to $75 \%$ of control over Train $_{10-20}$. There was no significant plasticity in 26 connections (12\%).

Depression was marginally the most frequent effect at $10 \mathrm{~Hz}$ (34\% depressed, 33\% unchanged, 18\% facilitated, 15\% biphasic; $n=134)$. Facilitation developed to $160 \%$ of control by the second spike, with modest recovery occurring again later in the train. Biphasic connections facilitated to $\sim 130 \%$ of control before depressing over Train $_{6-10}$ to reach a plateau of $70 \%$ of control.

At $5 \mathrm{~Hz}, 52 \%$ of connections depressed, 27\% were unchanged, $13 \%$ facilitated, and $8 \%$ were biphasic $(n=97)$. Peak facilitation to $150 \%$ of the initial EPSP was reached by the third spike in the train (Fig. 2 B). Biphasic connections facilitated to $110 \%$ of control before depressing over $\operatorname{Train}_{6-10}$ to reach depression of $60 \%$ of control.

The same type of plasticity was usually evoked when single connections were examined at each frequency ( $n=69$ of 95). Relatively consistent features in connections where the plasticity differed at different frequencies were facilitation at 10 and $20 \mathrm{~Hz}$ but depression or no plasticity at $5 \mathrm{~Hz}(n=6)$, and depression at $20 \mathrm{~Hz}$ but unchanged responses at 10 and $5 \mathrm{~Hz}(n=4)$.

Significant depression or facilitation occurred over Train $2-5$ $(p<0.05)$ (Fig. 2A,B). The plasticity could thus develop during network activity and influence the patterning of the network out- 
put (Buchanan and Cohen, 1982; Buchanan and Kasicki, 1995). Overall, where depression occurred the plateau level was significantly greater $(p<0.01)$ at $10 \mathrm{~Hz}(46 \pm 3 \%)$ than at 20 or $5 \mathrm{~Hz}$ (66 \pm 4 and $64 \% \pm 3 \%$ of control, respectively) (Fig. $2 A$ ). There was no frequency-dependent difference in the peak facilitation, although at $5 \mathrm{~Hz}$ it did not reach a stable plateau (Fig. 2B). The peak facilitation and depression at biphasic connections did not differ significantly at different frequencies ( $p>0.05$ ) (Fig. $2 C$ ).

The initial EPSP amplitude was significantly larger at depressing connections $(1.66 \pm 0.11 \mathrm{mV})$ than at facilitating $(1.08 \pm 0.1$ $\mathrm{mV})$, biphasic $(1.10 \pm 0.13 \mathrm{mV})$, or unchanged connections $(0.96 \pm 0.08 \mathrm{mV} ; p<0.05$; one-way ANOVA). Differences in EPSP rise times and half-widths were not associated with different forms of plasticity ( $p>0.05$; ANOVA). Activity-dependent changes in rise time and half-width were studied in 23 pairs in which EPSPs were free of stimulus artifacts. Because depression was the usual effect ( $n=18$ of 23), only depressing connections were examined. The half-width and rise time were usually unchanged over spike trains ( $n=14$ of 18 ), but in four connections the half-width was reduced at $20 \mathrm{~Hz}$ (data not shown).

\section{Convergence and divergence}

These results show that monosynaptic EIN-evoked EPSPs in motor neurons differ in their properties and plasticity. This is a common finding at spinal and supraspinal synapses (Koerber and Mendell, 1991; Markram et al., 1998; Thomson, 2000). The variability may reflect differences in presynaptic properties, for example, release probability or the available synaptic resources, or postsynaptic properties such as receptor desensitization or voltage-dependent dendritic conductances (see below). In spinal and supraspinal systems, convergent inputs onto single postsynaptic cells are often similar, whereas divergent outputs from single cells can differ in different postsynaptic targets (Koerber and Mendell, 1991; Markram et al., 1998). This suggests that the postsynaptic cell determines the presynaptic release properties. As an initial step in the analysis of the variability of EIN inputs, the properties of divergent and convergent connections were examined.

In a previous analysis using triple intracellular recordings, divergent inputs from a single EIN to two motor neurons were rare ( $n=2$ of 21) (Parker and Grillner, 2000). Although the number of triple recordings has been increased more than twofold, the proportion of EINs that made divergent connections onto both postsynaptic motor neurons was approximately the same $(n=5$ of 47 triple recordings). The initial EPSP amplitude evoked by a single EIN in two postsynaptic motor neurons could vary markedly (range, $0.37-2.5 \mathrm{mV}$ ), in one case by a factor of three. In three of the five divergent connections the plasticity during the train was similar in the two postsynaptic cells, but in two of five the initial EPSP amplitude and plasticity differed (data not shown). The small sample size makes it difficult to make definite conclusions about the properties of divergent connections.

In contrast to divergence, convergent EIN inputs from two or more EINs to a single motor neuron were common. Up to seven EINs could connect to a single motor neuron $(n=44)$. This does not necessarily reflect the maximum number of convergent inputs a motor neuron can receive, but the number found before a motor neuron recording was lost. There were varying patterns of convergent inputs. The initial EPSP amplitude and plasticity over the train could differ (Fig. 3Ai,Aii); both the initial EPSP amplitude and plasticity could be the same (data not shown); the initial EPSP amplitude could differ, but the plasticity over the train was the same (Fig. 3Bi,Bii); the initial EPSP amplitude was similar,

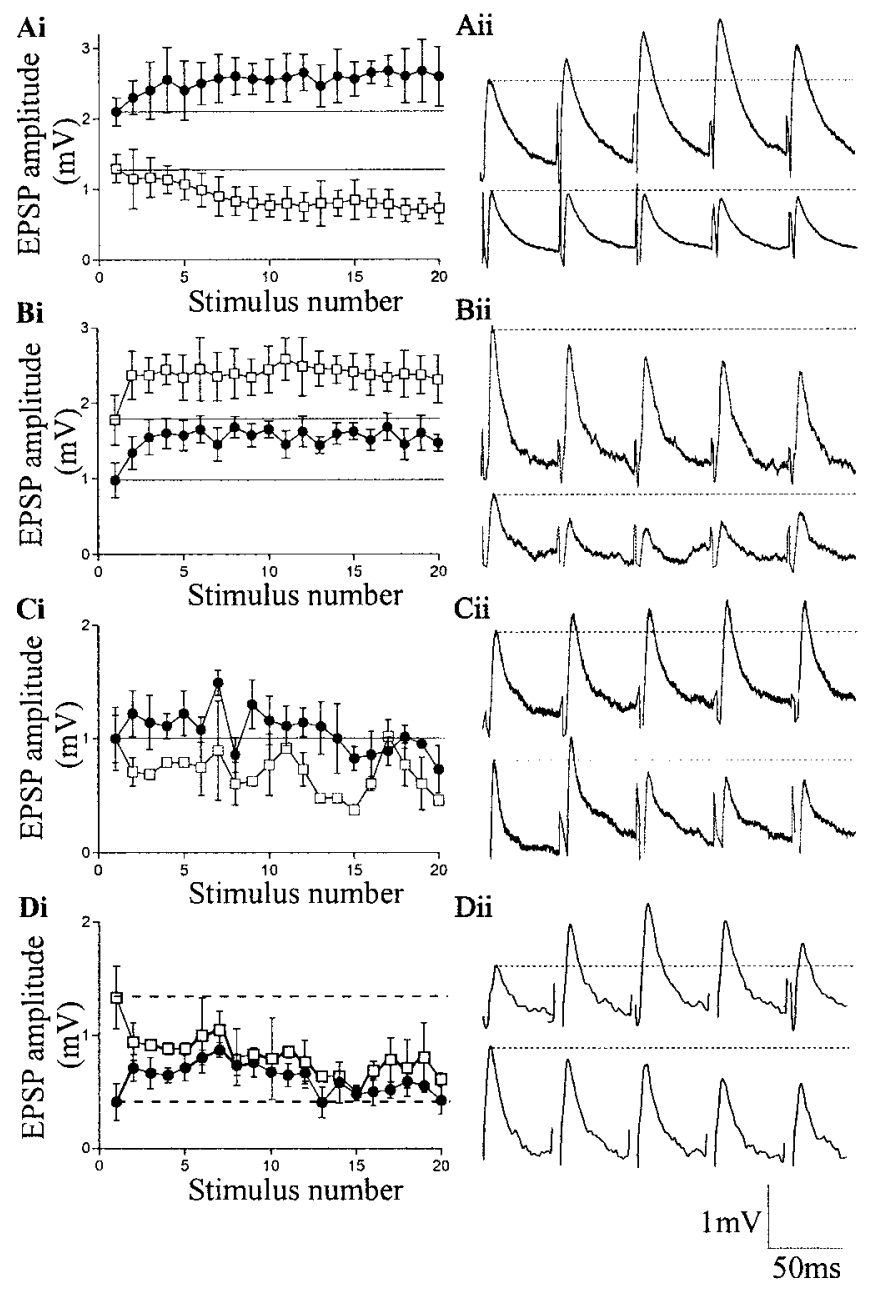

Figure 3. The properties of convergent EIN inputs to single motor neurons. The graphs and traces show inputs made by two different EINs onto a single motor neuron. Ai, Aii, Two convergent inputs to a single motor neuron in which the initial EPSP amplitude and plasticity differed. $B i$, Bii, Convergent inputs in which the same type of plasticity developed from different initial EPSP amplitudes. Ci, Cii, Convergent inputs that evoked different plasticity from two EPSPs with similar initial amplitudes. Di, Dii, Convergent inputs where EPSPs with different initial amplitudes and plasticity resulted in the input equalizing during the spike train.

but the plasticity differed (Fig. 3Ci,Cii); and both the initial EPSP amplitude and plasticity could differ, but in this case the different plasticity equalized the input during the spike train (Fig. 3Di, Dii).

The initial amplitude and plasticity of convergent EIN inputs to single motor neurons can thus vary. This suggests that the presynaptic EIN and not the postsynaptic motor neuron essentially determined the properties of the synapse. Importantly, the properties of convergent inputs to single motor neurons show that the synaptic variability is not simply related to differences between animals, experiments, or inputs to different classes of motor neurons (Rovainen, 1979; Wallén et al., 1985), but that it is an intrinsic property of the locomotor network.

\section{Locus of plasticity}

Although postsynaptic voltage, AMPA receptor desensitization, or properties or the presynaptic action potential do not contribute to the plasticity of EIN inputs (Parker, 2000b; and my unpublished observations), the locus of the plasticity is unknown. It has been investigated here by examining changes in the mean quantal content $(m)$ over PP responses using the inverse of the coefficient of variation $\left(m=1 / \mathrm{CV}^{2}\right)$ (Malinow and Tsien, 1990; Selig et al., 
A

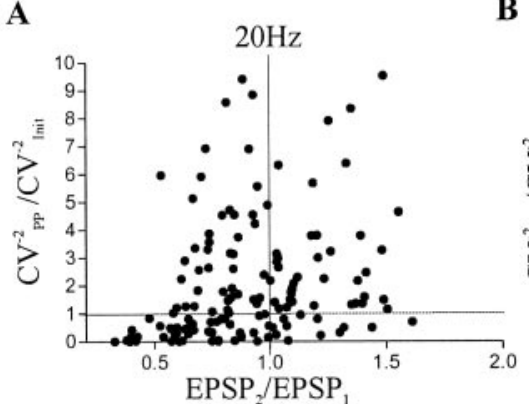

D

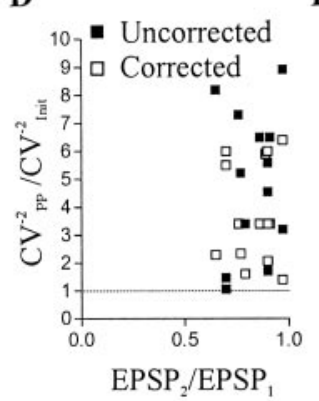

E

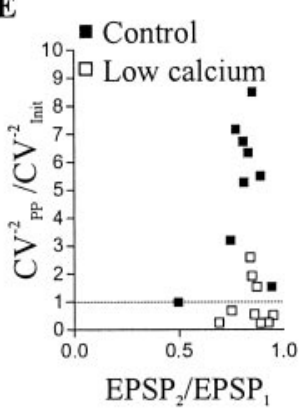

B

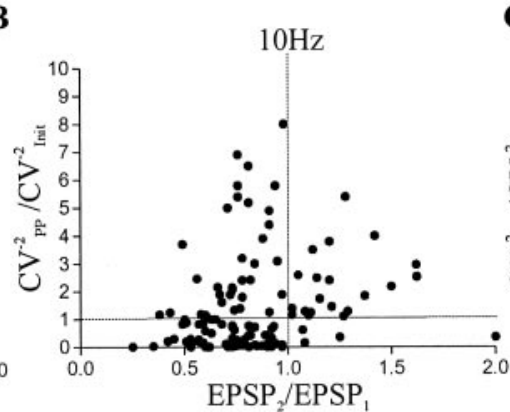

F

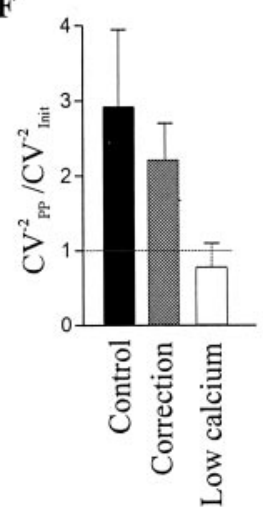

C

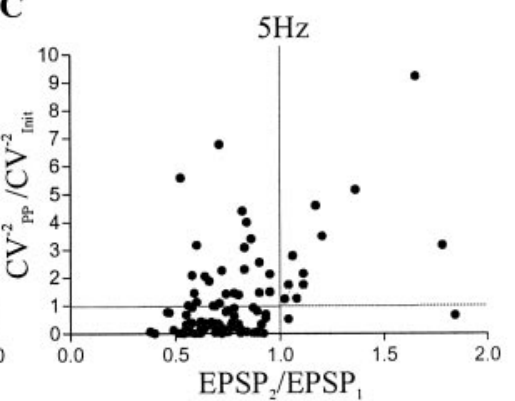

G
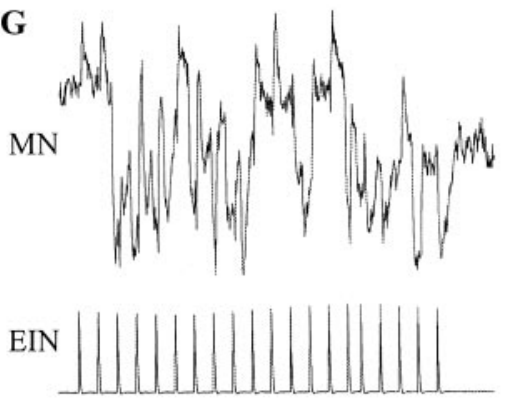

$1 \mathrm{mV}$

$40 \mathrm{mV}$

$200 \mathrm{~ms}$

Figure 4. Examination of the locus of plasticity using the $\mathrm{CV}^{-2}$. Graph showing the relationship of the $\mathrm{CV}^{-2}\left(\mathrm{CV}^{-2}{ }_{\mathrm{PP}} / \mathrm{CV}^{-2}\right.$ Init $)$ to paired pulse plasticity $\left(\mathrm{EPSP}_{2} / \mathrm{EPSP}_{1}\right)$ at $20 \mathrm{~Hz}(A), 10 \mathrm{~Hz}$ $(B)$, and $5 \mathrm{~Hz}(C)$. Each symbol represents a single connection. With a presynaptic mechanism the $\mathrm{CV}^{-2}{ }_{\mathrm{PP}} / \mathrm{CV}^{-2}$ Init should increase with facilitation but reduce with depression. As can be seen from the graphs, facilitation tended to follow a presynaptic mechanism. However, with depression the response was more variable, and at higher frequencies there was an increase in the number of responses in which the $\mathrm{CV}^{-2}{ }_{\mathrm{PP}} / \mathrm{CV}^{-2}$ Init increased with depression. The increase in the $\mathrm{CV}^{-2}$ with depression was not removed by correcting for the effect of driving force resulting from the initial EPSP (D) but was reduced in low-calcium Ringer's solution $(E) . F$, Graph showing the $\mathrm{CV}^{-2}{ }_{\mathrm{PP}} / \mathrm{CV}^{-2}$ Init at depressing connections in control, after correcting for driving force, and in low-calcium Ringer's solution. $G$, Example trace showing a significant polysynaptic inhibitory input evoked in a motor neuron by EIN stimulation.

1995). The $\mathrm{CV}^{-2}$ of the second EPSP should be reduced relative to the $\mathrm{CV}^{-2}$ of the initial EPSP $\left(\mathrm{EPSP}_{1}\right)$ if PP depression is caused by a presynaptic reduction of transmitter release (reduced $m$ ), but increased relative to the $\mathrm{CV}^{-2}$ of $\mathrm{EPSP}_{1}$ if $\mathrm{PP}$ facilitation is associated with increased transmitter release (increased $m$ ).

The PP CV ${ }^{-2}$ was increased relative to the control $\mathrm{CV}^{-2}$ in 38 of 46 facilitating connections at $20 \mathrm{~Hz}$ (83\%) (Fig. $4 \mathrm{~A}$ ), in 20 of 24 connections at $10 \mathrm{~Hz}(83 \%)$ (Fig. $4 B$ ), and in 11 of 13 connections at $5 \mathrm{~Hz}(85 \%)$ (Fig. 4C). These results essentially support a presynaptic increase in the mean quantal content $(m)$ during facilitation. At depressing connections, the $\mathrm{PP} \mathrm{CV}^{-2}$ was reduced relative to the control $\mathrm{CV}^{-2}$ in 43 of 85 connections (51\%) at $20 \mathrm{~Hz}$ (Fig. 4A), in 59 of 92 connections at $10 \mathrm{~Hz}$ (64\%) (Fig. $4 B$ ), and in 63 of $85(74 \%)$ connections at $5 \mathrm{~Hz}$ (Fig. 4C). Only $50 \%$ of the connections at $20 \mathrm{~Hz}$ thus showed the reduced $\mathrm{CV}^{-2}$ expected of presynaptic depression, although the proportion increased at lower stimulation frequencies.

An increased $\mathrm{CV}^{-2}$ would paradoxically suggest an increase in the mean quantal content during depression. However, it could also reflect a change in postsynaptic responsiveness (Malinow and Tsien, 1990). Depression is not associated with AMPA receptor desensitization (Parker, 2000b), and because the input depresses the reduced EPSP, variability cannot be accounted for by the saturation of postsynaptic receptors. These features suggest against a change in the properties of postsynaptic glutamate receptors. Depressing connections in which the $\mathrm{CV}^{-2}$ increased tended to have relatively large EPSP amplitudes (mean $1.96 \pm$ $0.53 \mathrm{mV}$ ). A larger postsynaptic conductance can reduce the variability of subsequent PSPs in a train, thus accounting for the increased $\mathrm{CV}^{-2}$ with depression (McLachlan and Martin, 1981).
The effect of the synaptic conductance was compensated for using the correction factor of McLachlan and Martin (1981). Assuming a quantal value of $0.12 \mathrm{mV}$ (see below), resting membrane potentials of between -60 and $-70 \mathrm{mV}$, and EPSP amplitudes of 3-4 $\mathrm{mV}$, the upper range of EIN-evoked inputs, the correction added $0.05-0.1 \mathrm{mV}$ to successive EPSPs in the train $(n=14)$. Although this reduced the $\mathrm{CV}^{-2}$, it was not sufficient to remove the increased $\mathrm{CV}^{-2}$ with depression (Fig. $4 D, F)$.

As synapses are examined in an intact network, evoked inputs can occur on a certain amount of background synaptic noise. In quantal analyses this noise reduces the EPSP variability and could thus also account for the increased $\mathrm{CV}^{-2}$ with depression. The baseline synaptic noise should be the same at all frequencies and thus would not account for the frequency dependence of the increased $\mathrm{CV}^{-2}$ with depression. In some cases, however, EIN stimulation resulted in frequency-dependent polysynaptic inhibitory and excitatory inputs (an example is shown in Fig. 4G). This presumably reflects the feedforward activation of neurons that converged onto the postsynaptic cell. These inputs would not be accounted for by the correction factor used above. Although connections with obvious polysynaptic inputs were not included in the analysis ( $n=26$ ) (my unpublished observations), it was possible that in some cases a reduced polysynaptic input was present that increased the background synaptic noise and affected the evoked EPSP. This possibility was examined using low-calcium Ringer's solution. This Ringer's solution reduced the amplitude of the evoked EPSP by $\sim 50 \%$ and blocked polysynaptic inputs (my unpublished observations), presumably by preventing EINevoked EPSPs from reaching spike threshold in interposed neu- 
A

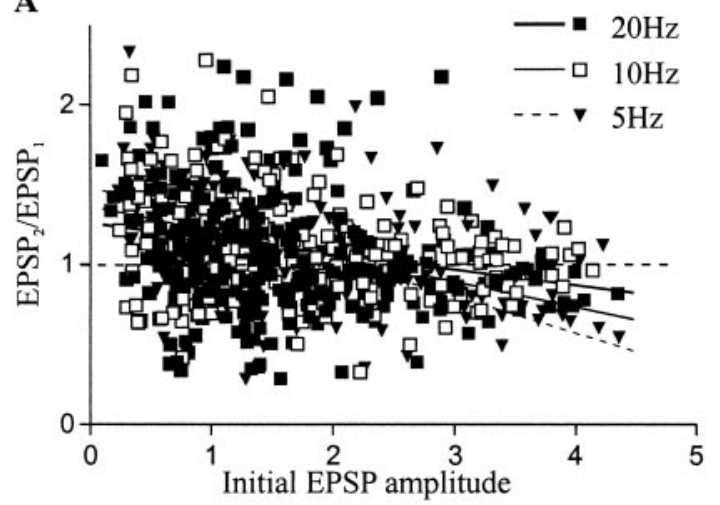

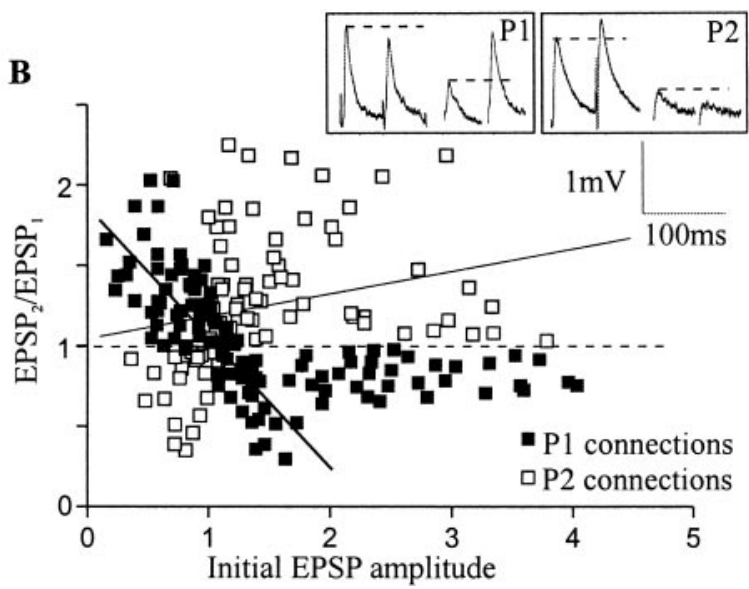

Figure 5. The relationship of PP plasticity (EPSP $/$ /EPSP $P_{1}$ ) to the initial EPSP amplitude. $A$, Graph showing PP plasticity at different frequencies. For clarity only PP plasticity with values of $<2.5$ is shown. $B$, The relationship of PP plasticity to the initial EPSP amplitude is shown at $20 \mathrm{~Hz}$, when connections that show an inverse relationship between initial EPSP amplitude and plasticity (P1 connections, $\square$ ) are plotted separately to connections that have PP plasticity positively related to EPSP amplitude (P2 connections, $\square$ ). Notice that above $2 \mathrm{mV}$ the EPSP was not related to PP plasticity in either type of connection. The inset shows sample traces of paired-pulse plasticity from P1 and P2 connections at $20 \mathrm{~Hz}$.

rons. Low-calcium Ringer's solution usually reduced the depression, and in some cases converted it into facilitation (Parker, 2000b). Only connections in which depression still occurred were examined. Low-calcium Ringer's solution reduced the number of connections in which the $\mathrm{CV}^{-2}$ was increased, and overall depression was associated with a reduction in the $\mathrm{CV}^{-2}(n=9)$ (Fig. 4E,F). This effect could not be accounted for by the reduced depression in low-calcium Ringer's solution, because this would increase the $\mathrm{CV}^{-2}$. It instead suggests that the depression was mediated presynaptically, but that polysynaptic inputs to the postsynaptic cell can influence the EPSP variability.

\section{The influence of the initial EPSP amplitude on plasticity}

The activity-dependent plasticity of EIN inputs thus appears to be mediated presynaptically. Presynaptic influences on plasticity are often associated with differences in the initial release probability (Zucker, 1989). There was previously a weak relationship between the initial EPSP amplitude (assumed to reflect release probability) and the plasticity of EIN inputs (Parker, 2000b). However, as with the plasticity over spike trains (see above), this relationship could have been influenced by variability in the small sample size. The influence of the initial EPSP amplitude on plasticity was thus reexamined.

Overall, plasticity was negatively related to the initial EPSP amplitude, the relationship expected of a release probabilitydependent mechanism (Fig. 5A) (Zucker and Regehr 2002). However, $r^{2}$ values were low at all frequencies and all parts of the train $(0.16 \pm 0.03$; range, $0.03-0.27)$, again suggesting a weak influence of the initial EPSP amplitude on plasticity (Waldeck et al., 2000).

The low $r^{2}$ values reflected the development of facilitation and depression from both small and large initial EPSPs. To examine further the relationship between the initial EPSP amplitude and plasticity, connections in which the plasticity was positively related to the EPSP amplitude (i.e., small depressing and large facilitating connections) were removed from the grouped data and plotted separately. Only PP responses were examined. At $20 \mathrm{~Hz}$, 110 of 252 pairs $(44 \%)$ were removed (Fig. $5 B$ ). Of these, 74 $(67 \%)$ were EPSPs of $>1 \mathrm{mV}$ that facilitated. The remainder were small $(<1 \mathrm{mV})$ depressing EPSPs. At $10 \mathrm{~Hz}, 86$ pairs were removed and plotted separately (44\%). Of these, 76 (88\%) were facilitating EPSPs of $>1 \mathrm{mV}$ (data not shown). At $5 \mathrm{~Hz}, 76$ pairs were removed and plotted separately (39\%), of which $69(91 \%)$ were large initial EPSPs that facilitated (data not shown).

Separating the connections in which plasticity was positively or negatively related to the initial EPSP amplitude not surprisingly resulted in higher $r^{2}$ values over the different regions of the train $(\mathrm{P} 1,0.28 \pm 0.03$, range, $0.16-0.42 ; \mathrm{P} 2,0.39 \pm 0.09$, range, $0.06-0.77)$. However, with EPSPs of $>2 \mathrm{mV}$ there was a reduced range of plasticity and little influence of the initial EPSP amplitude over a $2 \mathrm{mV}$ range in both groups (Fig. 5B). The different responses at connections in which plasticity was negatively or positively related to the initial EPSP amplitude presumably reflect differences in the synaptic properties at these connections. For convenience, in the subsequent analysis connections in which plasticity was negatively related to the initial EPSP amplitude (i.e., small facilitating and large depressing EPSPs) will be termed P1, and those in which there was a positive relationship (small depressing and large facilitating EPSPs) will be termed P2.

\section{Variance-mean analysis of synaptic properties}

The basic properties and plasticity of EIN-evoked EPSPs thus varied, suggesting that EINs do not form a functionally homogenous interneuron population. The plasticity appears to mostly be mediated presynaptically. Differences between connections should thus be reflected in differences in their presynaptic release properties. These properties are usually examined using a quantal analysis. However, this analysis can be difficult to apply at central synapses (Korn and Faber, 1992). Discrete peaks in histograms of EIN-evoked EPSP amplitudes indicative of quantal transmission were lacking (my unpublished data). This could reflect the influence of electrical or synaptic noise on EPSP measurements or the location of inputs at varying distances from the soma. A technique for examining synaptic parameters that is less sensitive to the effects of recording noise and makes fewer assumptions about synaptic properties has recently been introduced (Clements and Silver, 2000). This method examines the PSP variance and mean under conditions of altered release probability ( $\mathrm{V}-\mathrm{M}$ analysis). At low release probabilities fewer sites release transmitter, and the EPSP amplitude and variance are reduced. At high release probabilities almost all sites release transmitter. This increases the EPSP amplitude but again reduces the variance. At intermediate release probabilities the number of sites that release transmitter fluctuates from trial to trial. This results in intermediate EPSP 
amplitudes and an increased variance. The relationship of the variance to the mean at different release probabilities can be approximated by a parabola (Clements and Silver, 2000): $y=A x-B x^{2}$, where $y$ is the PSP variance and $x$ is the mean amplitude. $A$ and $B$ are free parameters that are adjusted to optimally fit the parabola to the $\mathrm{V}-\mathrm{M}$ data. The quantal amplitude $\left(q_{w}\right)$, release probability $\left(p_{w}\right)$, and number of release sites $\left(n_{\min }\right)$ can then be calculated as: $q w=A /\left(1+\mathrm{CV}^{2}\right), p w=x(B / A)(1+$ $\left.\mathrm{CV}^{2}\right)$, and $\mathrm{n}_{\min }=1 / B$, where $q_{w}$ and $p_{w}$ are weighted averages, i.e., they emphasize terminals with larger quantal amplitudes and release probabilities, and $n_{\min }$ is the minimum number of release sites.

Release probability was altered by changing Ringer's solution calcium levels or by using cadmium to block calcium entry. In the best cases $(n=3)$, two low (50 and $75 \%$ ) and two high (150 and 200\%) calcium Ringer's solutions could be used before a recording was lost, but usually only 50 and 200\% calcium Ringer's solution could be used $(n=26)$. At least 100 EIN-evoked EPSPs were evoked at a frequency of $0.2 \mathrm{~Hz}$ under each condition (Fig. 6A). Stimulation at this frequency did not usually evoke any plasticity (Fig. $6 B)$, but if this occurred $(n=3)$ the connection was not included in the analysis.

The mean quantal amplitude $\left(q_{w}\right)$ was $0.12 \pm 0.01 \mathrm{mV}$ (range, $0.06-0.22 \mathrm{mV} ; n=29$ ) (Fig. 6Di). This is less than half the amplitude of TTX-resistant miniature EPSPs (mEPSPs) (range, $0.3-0.55 \mathrm{mV}$ ) (Parker, 2000c). The discrepancy may reflect the difficulty in identifying mEPSPs of $\sim 0.1 \mathrm{mV}$ in the baseline recording noise. In addition, TTX-resistant mEPSPs are clearly seen in only a proportion of experiments (approximately one in seven) and could represent spontaneous release from a specific population of synapses. The minimum number of release sites $\left(n_{\text {min }}\right)$ was $25 \pm 3$ (range, $16-48 ; n=15$ ) (Fig. 6 Dii), and the release probability $\left(p_{w}\right)$ was $0.46 \pm 0.05$ (range, $0.32-0.73 ; n=$ 15) (Fig. 6Diii). These values of $n_{\min }$ and $p_{w}$ give a mean quantal content $\left(m=n_{\min } p_{w}\right)$ of 11 (range, 5-35). With a quantal amplitude of $0.12 \mathrm{mV}$, this value of $m$ gives a mean EPSP of $1.32 \mathrm{mV}$ (range, $0.6-4.2 \mathrm{mV}$ ). This approximates the mean and range of all evoked EPSPs (1.50 mV; range, $0.32-4.2 \mathrm{mV}$; see above).

The $p_{w}$ ranged from moderate $(0.32)$ to relatively high values (0.73). However, the evidence suggests a wider range of $p_{w}$ than this. First, in some cases the relationship of the EPSP mean to variance was linear $(n=14)$. In these cases only $q_{w}$ can be determined (Clements and Silver, 2000). Although $p_{w}$ cannot be calculated, a linear relationship reflects a low release probability $(<0.3)$ (Clements and Silver, 2000). Second, high-calcium Ringer's solution, which should increase the release probability, failed to increase the EIN-evoked EPSP amplitude in some connections $(n=9$ of 42). The lack of effect of high calcium on the EPSP amplitude suggests that the release probability was already high at these connections (see below) (Murthy et al., 1997) and presumably greater than the maximum $p_{w}$ of 0.73 obtained in the $\mathrm{V}-\mathrm{M}$ analysis.

To determine the role of synaptic properties in the plasticity of
EIN inputs, the $q_{w}, p_{w}$, and $n_{\text {min }}$ were compared at depressing and facilitating connections. The $q_{w}$ was $0.08 \pm 0.01 \mathrm{mV}$ at depressing connections and $0.10 \pm 0.04 \mathrm{mV}$ at facilitating connections (Fig. $6 \mathrm{Ei})$. The $n_{\min }$ was $23.5 \pm 2.1$ at depressing connections and $28.3 \pm 10$ at facilitating connections (Fig. 6 Eii). The $p_{w}$ was $0.67 \pm 0.1$ at depressing connections and $0.32 \pm 0.08$ at facilitating connections (Fig. 6Eiii). Only $p_{w}$ differed significantly between depressing and facilitating connections $(p<0.05)$, suggesting that, as in other systems (Zucker, 1989; Thomson, 2000), activity-dependent synaptic plasticity is influenced by the presynaptic release probability. Interestingly, high-calcium Ringer's solution had a smaller potentiating effect on the EPSP amplitude at depressing connections ( $126 \pm 11 \%$ of control) than at facilitating connections ( $247 \pm 19 \%$ of control; data not shown), supporting the suggestion above that the absence of a potentiating effect of high-calcium Ringer's solution on the EPSP amplitude reflects the influence of a high initial $p_{w}$.

For comparison with EIN-evoked EPSPs, glutamatergic inputs from reticulospinal axons to motor neurons were also examined using a V-M analysis (Fig. 6A,Di-Diii) $(n=9)$. The $q_{w}$ of reticulospinal inputs was $0.14 \pm 0.04 \mathrm{mV}$ (range, $0.03-0.23 ; n=$ 9). This is a mean and range similar to that of the EINs and thus suggests that reticulospinal axons are not the source of larger TTX-resistant mEPSPs (see above). The $p_{w}$ was less than the EINs $(0.38 \pm 0.025$; range, $0.33-0.49 ; n=6)$. In three connections the $\mathrm{V}-\mathrm{M}$ relationship was linear, again suggesting release probabilities of $<0.3$. However, high-calcium Ringer's solution potentiated reticulospinal-evoked EPSPs in every case ( $n=9$ of 9$)$, suggesting a reduced upper range of $p_{w}$ compared with the EINs. Finally, the mean number of release sites $\left(n_{\text {min }}\right)$ was greater than at the EINs ( $85 \pm 56$; range, $12-250 ; n=6$ ), and consequently the mean quantal content $\left(m=n_{\text {min }} p_{w}\right)$ was larger (32; range, 5-95). With a quantal amplitude of $0.14 \mathrm{mV}$, evoked EPSP amplitudes 


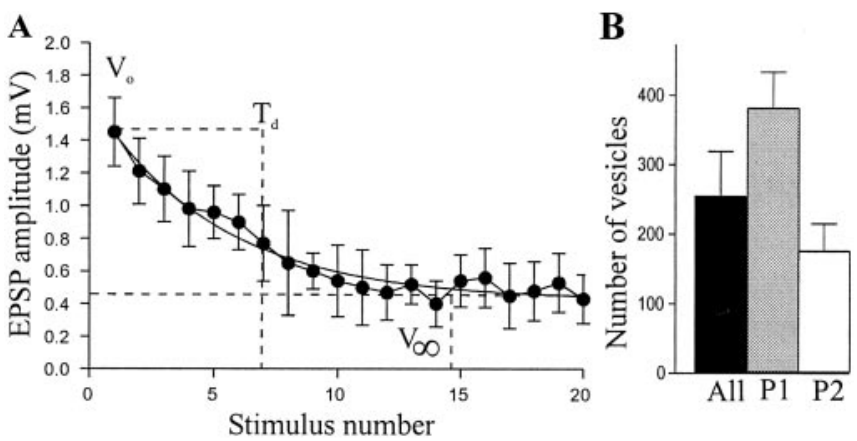

Figure 7. The releasable vesicle pool size in EINs. A, Graph showing the depression of an EIN-evoked EPSP in a single connection, illustrating the parameters used to estimate the available vesicle numbers. $B$, Histogram showing vesicle numbers in all depressing connections and in P1 and P2 connections. The estimated releasable vesicle pool was significantly larger at $P 1$ than at $P 2$ connections.

could theoretically range from 0.7 to $13.3 \mathrm{mV}$. This is a much greater range than EIN EPSPs. It is consistent with the observation that reticulospinal inputs typically have larger amplitudes than EIN-evoked EPSPs, although the theoretical upper range is greater than that observed experimentally (observed range, 0.57$8.5 \mathrm{mV}$ ) (my unpublished observations).

\section{Number of available synaptic vesicles}

The difference in $p_{w}$ at facilitating and depressing connections could account for the negative relationship between the initial EIN-evoked EPSP amplitude and plasticity at P1 connections. A high $p_{w}$ will release a large proportion of the available transmitter store. This will result in a large initial EPSP that could subsequently depress because of transmitter depletion. A low initial $p_{w}$ will release less transmitter. It will thus evoke a smaller initial EPSP but could provide the potential for a subsequent increase in $p_{w}$ and facilitation over the train (Zucker, 1989). Although consistent with the plasticity at P1 connections, release probability alone cannot account for the plasticity at P2 connections, where large initial EPSPs (presumed high release probability) facilitate and small initial EPSPs depress.

The release probability acts on or is influenced by the available synaptic resources (Schikorski and Stevens 1999; Zucker and Regehr, 2002). To examine whether differences in synaptic resources contributed to the variable properties of EIN-evoked EPSPs, the number of available synaptic vesicles was examined using the model of Wang and Zucker (1998). This model is based on the depression of synaptic inputs. It assumes two vesicle pools, available or unavailable. The available pool includes releaseready and reserve vesicles. Depression is assumed to be caused by the depletion of this pool. The number of vesicles in the available pool $\left(N_{v e s}\right)$ is given by: $V_{\mathrm{o}}{ }^{2} \tau d / q\left(V_{\mathrm{o}}-V \infty\right)$, where $V_{o}$ is the initial EPSP amplitude, $\tau d$ is the inverse rate constant of EPSP decay (expressed as the number of presynaptic spikes needed for the EPSP to drop to 1/e of the initial value), $q$ is the mean quantal amplitude, and $V \infty$ is the EPSP amplitude at the plateau level of depression (Fig. 7A).

With the quantal amplitude of $0.12 \mathrm{mV}$ estimated from the $\mathrm{V}-\mathrm{M}$ analysis, the mean number of available vesicles at these connections was $257 \pm 92$ (range, 34-954; $n=57$ ) (Fig. 7B). There was no significant difference between the number of available vesicles calculated from depression at 5,10 , or $20 \mathrm{~Hz}(p>$ 0.05; one-way ANOVA). This is expected, because the stimulation frequency should not affect basal vesicle numbers. However, the model assumes the absence of activity-dependent replenish- ment, which may not be the case at connections between EINs and motor neurons (Parker, 2000b). Activity-dependent replenishment will reduce the plateau level of depression $(V \infty)$ caused by depletion, thus resulting in an overestimation of the number of available vesicles. Whereas there was no overall difference in vesicle numbers at different frequencies, the estimated number of vesicles was greatest at $20 \mathrm{~Hz}$ in nine pairs (data not shown), which could reflect the influence of activity-dependent replenishment (Parker, 2000b).

The number of available vesicles varied 30 -fold between different connections. To determine whether this variability was related to functional differences in synaptic properties, the number of vesicles was compared at depressing $\mathrm{P} 1$ and $\mathrm{P} 2$ connections (Fig. $7 B$ ). Depressing P1 connections had significantly more vesicles ( $374 \pm 70 ; p<0.05 ; n=38$ ) than depressing P2 connections $(175 \pm 33 ; n=19)$. Although there was a twofold difference in the size of the available vesicle pool, in connections where a V-M analysis was also performed the $p_{w}$ was similar at both types of connection (P1, depressing $p_{w}=0.63, n=3 ; \mathrm{P} 2$, depressing $p_{w}=$ $0.56, n=4)$.

\section{Discussion}

This study shows variable properties at an individual synapse in the lamprey spinal cord. The variability occurred at convergent inputs onto single motor neurons and is thus an intrinsic network property.

Small sample sizes in previous analyses prevented synaptic variability from being examined. Large sample sizes are thus required if synaptic properties are to be examined in detail. Although possible at interneuron connections onto motor neurons, this will be difficult at connections between network interneurons where stable recordings are rare.

The analysis has been performed only in quiescent preparations. It is possible that activity-dependent synaptic properties are altered during network activity, for example, as a result of rhythmic presynaptic inputs (Alford et al., 1991). At some point, activity-dependent synaptic plasticity will have to be examined during network activity. However, these experiments are complicated by the difficulty in separating the synapse under study from other convergent inputs onto the postsynaptic cell (my unpublished observations).

\section{EPSP properties and plasticity}

EIN-evoked EPSPs are reliable and essentially never fail. However, EPSP amplitudes, rise times, half-widths, and plasticity differed between connections. These properties were weakly correlated, suggesting a potential continuum of functional properties that could influence the strength and integration of synaptic inputs. Further variability is suggested by the presence of electrical components in some connections and the ability of some EINs to reliably evoke postsynaptic spikes.

For plasticity to influence network activity it must occur over Train $_{2-5}$ (Buchanan and Cohen, 1982; Buchanan and Kasicki., 1995). Although its onset could vary between connections, significant depression or facilitation usually occurred over pairedpulse responses (Fig. 2A,B) and could thus influence the patterning of the network output. The early development of plasticity in this study contrasts with the previous analysis in which significant plasticity in the grouped data occurred only over Train ${ }_{6-10}$. The delayed onset to plasticity in the previous analysis presumably reflected the influence of variability in the small data set (Parker and Grillner, 1999). 


\section{Plasticity mechanisms}

Depression and facilitation could develop from small and large initial EPSPs. Plasticity could thus be negatively or positively related to the initial EPSP amplitude (P1 and P2 connections, respectively). Despite the weak relationship between the initial EPSP amplitude and plasticity in the grouped data, release probability could account for the properties of $\mathrm{P} 1$ and $\mathrm{P} 2$ connections.

The measured $p_{w}$ ranged from 0.3 to 0.73 , although there is evidence to suggest that it extends above and below this range (Hanse and Gustafsson, 2001). As in many systems (Zucker, 1989; Thomson, 2000), the $p_{w}$ was larger at depressing than at facilitating connections. This could account for the negative relationship between the initial EPSP amplitude and plasticity at P1 connections: a high release probability will evoke a larger initial EPSP that subsequently depresses, possibly caused by depletion, whereas a low release probability will evoke a smaller initial EPSP that has the potential to facilitate (Zucker, 1989; Thomson, 2000).

Release probability alone cannot account for the positive relationship between the EPSP amplitude and plasticity at P2 connections, where facilitating (presumed low release probability) connections have large initial EPSPs and depressing (presumed high release probability) connections have small initial EPSPs. The difference between P1 and P2 connections appears to reflect an interaction between release probability and the available transmitter store. The size of the available vesicle pool was examined using the model of Wang and Zucker (1998). This model could be applied only to depressing connections. Although Wang and Zucker (1998) applied it to the depression that followed initial facilitation, this was not possible here, because facilitation does not decay into depression over the trains used. The model also assumes that release and replenishment occur at constant rates. This is probably not valid for the EINs, because replenishment is activity and calcium dependent (Parker, 2000b). This replenishment mechanism could reduce depression during spike trains and thus result in an overestimation of the vesicle pool size. Despite these caveats, the estimated vesicle pool obtained using this model could account for some of the differences between P1 and $\mathrm{P} 2$ connections. $\mathrm{P} 1$ connections had a larger available vesicle pool than P2 connections. The size of the available vesicle pool has been suggested to influence the release probability (Schikorski and Stevens 1999) (but see Xu-Friedman et al., 2001; Millar et al., 2002). However, the $p_{w}$ was similar at depressing P1 and P2 connections. The twofold difference in the available vesicle pool but similar $p_{w}$ at P1 and P2 connections suggests that pool size does not influence the EIN release probability. Assuming that $p_{w}$ reflects the release of a proportion of the available transmitter store (Zucker and Regehr, 2002), the large proportion of vesicles released on the initial EPSP at depressing P1 and P2 connections could deplete the pool and cause subsequent depression. However, the difference in the size of the available vesicle pool means that depression will develop from a larger initial EPSP at P1 connections than at P2 connections. A similar effect could account for the facilitation at P1 and P2 connections, but in this case P2 connections, where the initial EPSP amplitude is relatively large, would be expected to have the larger vesicle pool.

\section{Interacting influences on network synaptic transmission}

An interaction between the release probability and the available vesicle pool thus presynaptically influences the EPSP amplitude and plasticity. There is also evidence that suggests that postsynaptic properties could also influence the integration of synaptic inputs. For example, the $\mathrm{CV}^{-2}$ analysis suggests that synaptic noise evoked by polysynaptic inputs during spike trains influenced the synaptic variability. This could be equivalent to stochastic resonance and may increase signal detection or reduce the influence of the location of the synaptic inputs (Stacey and Durand, 2001). The ability of some EIN inputs to evoke spikes also appears to reflect a postsynaptic property.

Network interneurons generate repetitive bursts of spikes during swimming. As a result, the replenishment of transmitter stores will also influence transmission over repetitive spike bursts. The summed depolarization over a train of five spikes, the upper number of spikes during a locomotor burst (Buchanan and Cohen, 1982; Buchanan and Kasicki, 1995), is $\sim 5.8 \mathrm{mV}$ at depressing connections and $8.5 \mathrm{mV}$ at facilitating connections. Over the range of quantal amplitudes determined here (0.06$0.22 \mathrm{mV})$, this requires the release of between 26 and 97 vesicles (10-38\% of the mean available vesicle pool) at depressing connections and between 39 and 142 vesicles (15-55\% of the mean pool) at facilitating connections. A large proportion of the vesicle pool could thus be released during a single locomotor burst, and without replenishment total depletion will occur after several bursts or within $\sim 10 \mathrm{sec}$ at low burst frequencies. This depletion is prevented by an activity- and calcium-dependent replenishment mechanism that is activated over Train ${ }_{2-5}$ (Parker, 2000b; and my unpublished observations).

Presynaptic influences during an episode of network activity will thus reflect an interaction between the release probability, the size of the available transmitter store, and the efficiency of transmitter replenishment. For example, decreasing the release probability by modulating calcium entry will reduce the initial EPSP amplitude and consequently reduce depression caused by depletion. Increasing the release probability could release a larger proportion of the available transmitter store, which will result in an increase in the initial EPSP amplitude and enhance depression caused by depletion. Because replenishment appears to be coupled to calcium entry and transmitter release (Parker, 2000b), it could maintain transmission at a set level by compensating for variations in the proportion of transmitter released from the available store. However, changing the ratio of release to replenishment will affect the size of the available transmitter store and thus alter synaptic properties over spike bursts. For example, increasing calcium entry will have little effect on connections with high initial release probabilities, but if calcium-dependent replenishment were enhanced, the size of the available transmitter pool would be increased, and a depressing P2 connection would be converted into a depressing P1 connection.

\section{Function of interneuron variability}

Synaptic properties vary at synapses made between different classes of interneurons in the lamprey spinal cord (Parker, 2000a). This is a common property of network synapses (Thomson, 2000). Variability has now also been shown at a single class of synapse. EIN inputs to motor neurons ranged from functionally strong (reliably evoked postsynaptic spikes) to weak (small depressing EPSPs). Function thus cannot be inferred from the anatomy, axonal projections, or transmitter content of interneurons (Gupta et al., 2000; McBain and Fisahn, 2001). The variability could reflect the modulation of properties in a homogenous interneuron pool or a heterogeneous population of interneurons with different functional roles.

The properties of synapses made between different classes of network interneurons could contribute to the specific role that these neurons have in patterning network activity. The variability at individual connections could instead provide an intrinsic 
mechanism for modifying the network output (Aradi and Soltesz, 2002). For example, the selection of different functional types of EINs will alter the excitatory drive to the network and thus modify the network output. The variation in EIN synaptic properties could also contribute to state-dependent influences on the plasticity of EIN transmission. Mechanisms that increase the release probability will have little effect on the initial EPSP amplitude at connections where the release probability is already high, but by influencing the available synaptic resources, the input during spike bursts could be altered. An effect of this sort could provide a basis for the metaplasticity of EIN-evoked synaptic transmission (Parker, 2000a).

\section{References}

Alford S, Christenson J, Grillner S (1991) Presynaptic GABAA and GABAB receptor-mediated phasic modulation in axons of spinal motor interneurons. Eur J Neurosci 3:107-117.

Aradi I, Soltesz I (2002) Modulation of network behaviour by changes in variance in interneuronal properties. J Physiol (Lond) 538:227-251.

Berry M, Pentreath V (1976) Criteria for distinguishing between monosynaptic and polysynaptic transmission. Brain Res 105:1-20.

Buchanan J (1993) Electrophysiological properties of identified classes of lamprey spinal neurons. J Neurophysiol 70:2313-2325.

Buchanan J, Kasicki S (1995) Activities of spinal neurons during brain stemdependent fictive swimming in lamprey. J Neurophysiol 73:80-87.

Buchanan JT (2001) Contributions of identifiable neurons and neuron classes to lamprey vertebrate neurobiology. Prog Neurobiol 63:441-466.

Buchanan JT, Cohen AH (1982) Activities of identified interneurons, motoneurons, and muscle fibers during fictive swimming in the lamprey and effects of reticulospinal and dorsal cell stimulation. J Neurophysiol 47:948-960.

Byrne J, Kandel E (1996) Presynaptic facilitation revisited: state and time dependence. J Neurosci 15:425-435.

Clements J, Silver R (2000) Unveiling synaptic plasticity: a new graphical and analytical approach. Trends Neurosci 23:105-113.

Dale N, Grillner S (1986) Dual-component synaptic potentials in the lamprey mediated by excitatory amino acid receptors. J Neurosci 6:2653-2661.

Feldman DE, Nicoll RA, Malenka RC (1999) Synaptic plasticity at thalamocortical synapses in developing rat somatosensory cortex: LTP, LTD, and silent synapses. J Neurobiol 41:92-101.

Fortune E, Rose G (2001) Short-term synaptic plasticity as a temporal filter. Trends Neurosci 24:381-385.

Getting P (1989) Emerging principles governing the operation of neural networks. Annu Rev Neurosci 12:185-204.

Gupta A, Wang Y, Markram H (2000) Organizing principles for a diversity of GABAergic interneurons and synapses in the neocortex. Science 287:273-278.

Hanse E, Gustafsson B (2001) Paired-pulse plasticity at the single release site level: an experimental and computational study. J Neurosci 21:8362-8369.

Juttner R, Meier J, Grantyn R (2001) Slow IPSC kinetics, low levels of alphal subunit expression and paired-pulse depression are distinct properties of neonatal inhibitory GABAergic synaptic connections in the mouse superior colliculus. Eur J Neurosci 13:2088-2098.

Koerber H, Mendell L (1991) Modulation of synaptic transmission at 1aafferent fiber connections on motorneurons during high-frequency stimulation: role of the postsynaptic target. J Neurophysiol 65:590-597.

Korn H, Faber D (1992) Quantal analysis and synaptic efficacy in the CNS. Trends Neurosci 14:439-445.

Malenka R, Nicoll R (1999) Long-term potentiation—a decade of progress? Science 285:1870-1874.
Malinow R, Tsien R (1990) Presynaptic enhancement shown by whole-cell recordings of long-term potentiation in hippocampal slices. Nature 346:177-180.

Marder E, Calabrese R (1996) Principles of rhythmic motor pattern generation. Physiol Rev 76:687-717.

Markram H, Wang Y, Tsodyks M (1998) Differential signaling via the same axon of neocortical pyramidal neurons. Proc Natl Acad Sci USA 95:5323-5328.

McBain C, Fisahn A (2001) Interneurons unbound. Nat Rev Neurosci 2:11-23.

McLachlan E, Martin A (1981) Non-linear summation of frog end-plate potentials in the frog and mouse. J Physiol (Lond) 311:307-324.

Millar AG, Bradacs H, Charlton MP, Atwood HL (2002) Inverse relationship between release probability and readily releasable vesicles in depressing and facilitating synapses. J Neurosci 22:9661-9667.

Murthy V, Sejnowski T, Stevens C (1997) Heterogenous release properties of visualized individual hippocampal synapses. Neuron 18:599-612.

Nadim F, Manor Y (2000) The role of short-term synaptic dynamics in motor control. Curr Opin Neurobiol 10:683-690.

O’Donovan M, Rinzel J (1997) Synaptic depression: a dynamic regulator of synaptic communication with varied functional roles. Trends Neurosci 20:431-433.

Parker D (2000a) Spinal cord plasticity: independent and interactive effects of neuromodulator and activity-dependent plasticity. Mol Neurobiol 21:1-26.

Parker D (2000b) Activity and calcium-dependent mechanisms maintain excitatory synaptic transmission in a rhythmic network. J Neurosci 20:1754-1766.

Parker D (2000c) Presynaptic and interactive peptidergic modulation of reticulospinal synaptic inputs in the lamprey. J Neurophysiol 83:2497-2507.

Parker D, Grillner S (1999) Activity-dependent metaplasticity of inhibitory and excitatory synaptic transmission in the lamprey spinal cord locomotor network. J Neurosci 19:1647-1656.

Parker D, Grillner S (2000) The activity-dependent plasticity of segmental and intersegmental synaptic connections in the lamprey spinal cord. Eur J Neurosci 12:2135-2146.

Rovainen C (1979) Neurobiology of lampreys. Physiol Rev 59:1007-1077.

Schikorski T, Stevens CF (1999) Quantitative fine-structural analysis of olfactory cortical synapses. Proc Natl Acad Sci USA 96:4107-4112.

Selig D, Hjelmstad G, Herron C, Nicoll R, Malenka R (1995) Independent mechanisms for long-term depression of AMPA and NMDA responses. Neuron 15:417-426.

Selverston A (1980) Are central pattern generators understandable? Behav Brain Sci 3:535-571.

Stacey W, Durand D (2001) Synaptic noise improves detection of subthreshold signals in hippocampal CAl neurons. J Neurophysiol 86:1104-1112.

Thomson A (2000) Molecular frequency filters at central synapses. Prog Neurobiol 62:159-196.

Waldeck RF, Pereda A, Faber D (2000) Properties and plasticity of pairedpulse depression at a central synapse. J Neurosci 20:5312-5320.

Wallén P, Grillner S, Feldman J, Bergelt S (1985) Dorsal and ventral myotome motoneurons and their input during fictive locomotion in lamprey. J Neurosci 5:654-661.

Wang C, Zucker R (1998) Regulation of synaptic vesicle recycling by calcium and serotonin. Neuron 21:155-167.

Xu-Friedman M, Harris K, Regehr W (2001) Three-dimensional comparison of ultrastructural characteristics at depressing and facilitating synapses onto cerebellar Purkinje cells. J Neurosci 21:6666-6672.

Zucker R (1989) Short-term synaptic plasticity. Annu Rev Neurosci 12:13-31.

Zucker RS, Regehr WG (2002) Short-term synaptic plasticity. Annu Rev Physiol 64:355-405. 